\title{
SEDA: Sistema para Exploração de Diretrizes de Acessibilidade
}

\author{
Guilherme Augusto A. Martins, Dilvan A. Moreira, Renata P. M. Fortes \\ ${ }^{1}$ Instituto de Ciências Matemáticas e de Computação \\ Universidade de São Paulo (USP) - São Carlos, SP - Brasil. \\ gaamart@icmc.usp.br, dilvan@icmc.usp.br, renata@icmc.usp.br
}

\begin{abstract}
Today, millions of individuals around the world face daily difficulties due to the lack of accessible information resources. Having in mind this problem, several sets of accessibility guidelines were created in order to generate recommendations for creating software and Web content that is more accessible. However, these sets of guidelines are usually related to generic disability definitions and many times it is difficult identify which guidelines are more adequate to particular disability cases. This paper proposes the use of a system, called SEDA (System for the Exploration of Accessibility Guidelines), to assist in identifying deficiencies for which no specific accessibility guidelines were created and existing limits when associating accessibility guidelines to deficiencies. We also present results of a case study based on a prototype, showing that the system can help researchers exploring accessibility guidelines issues and pointing out problems with the World Health Organization disability ontology, when there is a limited number of information items related to disabilities during the visualization.
\end{abstract}

Resumo. Atualmente milhões de indivíduos em todo o mundo enfrentam diariamente dificuldades devido à falta de recursos acessiveis para obterem informações. Tendo em mente esse problema foram criados diversos conjuntos de diretrizes de acessibilidade com o intuito de orientar, por meio de recomendações, a criação de programas e conteúdo Web mais acessíveis. Entretanto, tais conjuntos de diretrizes geralmente estão relacionadas com definições genéricas de deficiências e muitas vezes é difícil identificar quais diretrizes são mais adequadas para certos tipos de deficiências. Este trabalho propõe o uso do sistema denominado SEDA (Sistema para Exploração de Diretrizes de Acessibilidade) para auxiliar tanto na identificação de deficiências, para as quais não foram criadas diretrizes de acessibilidade específicas, quanto na identificação dos limites existentes ao se associar diretrizes de acessibilidade a deficiências. Nós também apresentaremos resultados de um estudo de caso baseado em um protótipo, demonstrando que o sistema pode auxiliar pesquisadores na exploração de diretrizes de acessibilidade e apontando alguns problemas na ontologia de deficiências da Organização Mundial de Saúde, quando se limita a quantidade de itens de informação relativos a deficiências na visualização.

\section{Introdução}

Atualmente no Brasil, existem mais de 45 milhões de pessoas com deficiências, em outras palavras $23,9 \%$ do total da população [IBGE 2010]. No mundo, esse número 
aumenta para 650 milhões de pessoas, o que representa quase $10 \%$ da população mundial [NU 2013]. Esses indivíduos enfrentam diariamente dificuldades devido à falta de recursos acessíveis para obterem informações ou utilizarem um dispositivo computacional. Eles precisam de apoio para exercer suas tarefas plenamente.

Diante dessa necessidade, foram criados diversos conjuntos de diretrizes de acessibilidade, como por exemplo a WCAG (Web Content Accessibility Guidelines) [W3C 2013] e a Section 508 ${ }^{1}$, com o intuito de orientar, por meio de recomendações, a criação de um conteúdo Web mais acessível. Assim, seguindo essas diretrizes, o desenvolvedor aumenta as chances de que o conteúdo Web criado estará disponível a todos os usuários.

Analisando-se o conjunto de diretrizes do WCAG, porém, observa-se que não existe uma organização adequada que permita classificá-las pelas diferentes deficiências citadas. Isso dificulta o trabalho de pesquisadores que queiram priorizar mecanismos que implementem soluções para um conjunto específico de diretrizes, conjunto esse que estaria mais relacionado a uma determinada deficiência.

Com essa questão em mente, Brajnik [2013] realizou o trabalho que, além de associar as diretrizes de acessibilidade da WCAG com as deficiências até então citadas, acrescentou novas categorias de usuários, como aqueles que utilizam o navegador com o Javascript desabilitado ou mesmo os que possuem epilepsia. Entretanto, tal abordagem visa apenas à classificação e não a exploração de novas diretrizes de acessibilidade com base nas deficiências.

Portanto, com base na busca por classificar diretrizes de acessibilidade utilizando os conceitos das deficiências e na utilização de informações estruturadas por meio de ontologias, foi proposto o SEDA, um sistema para apoiar a exploração de diretrizes de acessibilidade. Ele visa auxiliar os usuários tanto na identificação de deficiências, para as quais foram criadas diretrizes de acessibilidade, quanto para identificar os limites existentes ao realizar uma classificação entre deficiências e diretrizes.

Uma vez que, a princípio, os usuários do sistema SEDA serão prioritariamente especialistas da área de acessibilidade, que geralmente não possuem conhecimentos aprofundados sobre o uso de ferramentas para uso de ontologias (por exemplo, o Protégé [Protégé 2013]), foi proposta uma interface Web utilizando HTML5 e Javascript, com objetos JSON (JavaScript Object Notation) armazenados localmente no agente de usuário, para essa tarefa. Dessa maneira, é possível propiciar portabilidade e simplicidade na interação, uma vez que nenhuma outra ferramenta é necessária.

Ao final da interação do especialista com o sistema SEDA, ele obterá, como resultado, um relatório resumido, no formato HTML, contendo as respectivas associações realizadas e suas justificativas, assim como as justificativas encontradas nos requisitos de acessibilidade da Section 508, juntamente com as deficiências não associadas.

\footnotetext{
${ }^{1} \mathrm{http}: / / w w w . s e c t i o n 508 . g o v /$ 
O sistema SEDA utiliza uma ontologia proposta pela Organização Mundial da Saúde (OMS) para classificação de deficiências, a CIF (Classificação Internacional de Funcionalidade), cujo objetivo é proporcionar uma codificação ampla de informações sobre saúde (e.g. diagnósticos, funcionalidades e incapacidades) utilizando uma linguagem estruturada [OMS 2013]. De modo geral, o sistema permite que o usuário adicione qualquer conjunto de diretrizes, desde que disponibilizado em formato JSON. No estudo de caso, realizado como prova de conceito sobre a usabilidade do protótipo do sistema SEDA, foi utilizada uma ontologia criada pelo projeto Accessible, que visa explorar as ferramentas de acessibilidade e metodologias de normalização a fim de apoiar a produção de softwares acessíveis, devido ao fato de conter as informações presentes no conjunto de diretrizes propostas pela WCAG 2.0 [Accessible 2013], ou seja, além das diretrizes de acessibilidade, também estão os critérios de sucesso dos princípios de acessibilidade.

Este artigo está organizado em oito seções. A Seção 2 descreve a motivação para a concepção do sistema SEDA. A Seção 3 apresenta um breve referencial teórico dos principais temas abordados no trabalho. Na Seção 4 apresentamos a arquitetura do sistema. A Seção 5 apresenta o método de pesquisa utilizado para a realização do estudo de caso. A Seção 6 descreve em detalhes o estudo de caso que foi realizado, utilizando o protótipo do sistema SEDA para avaliar seu potencial de uso. Na Seção 7, é apresentada uma discussão sobre os resultados do estudo de caso. E, finalmente, na Seção 8, são apresentadas as conclusões sobre o trabalho realizado e alguns trabalhos futuros.

\section{Motivação para o sistema SEDA}

Diretrizes, geralmente, são definidas visando orientar pessoas, por meio de regras gerais a serem seguidas, quando existem muitas maneiras de se implementar soluções. Assim, em geral, as diretrizes podem ser definidas por meio de duas abordagens:

- A partir de estudos teóricos e gerais sobre as soluções gerais, que podem ser adotadas como possíveis soluções de problemas, ou;

- A partir de observações da realidade do espaço de problemas já existentes, que podem ser generalizados para serem descritos e servirem de objetivo para possíveis novas soluções.

Assim também ocorre com as diretrizes de acessibilidade. $\mathrm{Na}$ primeira abordagem, tem-se a elaboração de diretrizes inicialmente hipotéticas que geralmente necessitam de: um foco em um determinado componente da interface Web e um grupo de usuários, a implementação de uma nova funcionalidade visando resolver o problema identificado como uma barreira de acessibilidade, e, finalmente, a realização de testes com usuários com o intuito de validar as diretrizes propostas inicialmente [Jin et al. 2007] [Tsai and Lee 2009] [Watanabe et al. 2011] [Lee et al. 2009].

Já a segunda abordagem, mais experimental e iniciada pela observação da prática, ocorre, na maioria dos casos, o processo inverso ao descrito anteriormente [Iwase and Murata 2002]. Portanto, inicialmente são realizados diversos testes controlados com grupos de usuários específicos, no intuito de identificar possíveis problemas de acessibilidade, em seguida é realizado um levantamento dos problemas encontrados e então são elaboradas diretrizes para tais problemas, que poderão guiar novas implementações para resolver esses problemas. 
Entretanto, no âmbito das diretrizes de acessibilidade existentes, ambas as abordagens tratam as deficiências de modo geral, considerando as características apresentadas pelos usuários com quaisquer tipos de deficiência. Portanto, torna-se difícil que determinados nichos de usuários, apesar de estarem incluídos como pessoas com as deficiências tratadas genericamente, sejam identificados ou priorizados durante $\mathrm{o}$ desenvolvimento de aplicações Web mais dedicadas, pois as diretrizes propostas não são detalhadas o suficiente para satisfazer suas necessidades.

De fato, infelizmente não é possível atingir todos os nichos de usuários com uma determinada deficiência, com um conjunto restrito de diretrizes de acessibilidade. Entretanto, ignorar tais usuários fere o princípio do acesso universal a informação desejada na Internet [Ocenasek 2009]. Assim, é importante conhecer os limites existentes para as diretrizes de acessibilidade para que, ao menos, nos trabalhos futuros tais problemas possam ser abordados.

O sistema SEDA pode ajudar os pesquisadores de diretrizes, pois possibilita que eles realizem uma rápida classificação entre diretrizes propostas e um conjunto de deficiências específico, permitindo ao pesquisador identificar pontos a serem melhorados em sua pesquisa, por meio de uma visão mais ampla a respeito das deficiências e suas implicações.

Além disso, também pode auxiliar o usuário por meio da organização simples das informações, pela flexibilidade dos dados de entrada e pela sumarização dos resultados após todo o trabalho ser realizado. Devido a essas características, o sistema SEDA pode tornar o trabalho mais ágil e organizado e permite que o usuário possa se dedicar à tarefa relacionada com a correta classificação das diretrizes.

\section{A Acessibilidade Web e as Ontologias}

A Web foi concebida com o intuito de prover uma tecnologia para disponibilização de conteúdo em um formato padrão, simples e poderoso utilizando HTML [W3C, 1999], permitindo aos seus usuários acessarem uma grande variedade de informações e serviços. Entretanto, apesar de inventor da Web e diretor do W3C, Tim Berners Lee destacar que "O poder da Web está em sua universalidade", atualmente nem todas as pessoas podem usufruir da rede.

Thatcher et al. [2003] postulam que a acessibilidade deve incorporar a ideia de que todos os indivíduos têm o direito de serem incluídos na sociedade, independente de deficiências, localização geográfica, barreiras de linguagem, entre outros. Com isso, é possível definir a acessibilidade na Web como a possibilidade de qualquer usuário, utilizando qualquer agente de software ou hardware consiga entender e interagir com esse conteúdo.

Com o intuito de garantir essa acessibilidade, foram propostos diversos conjuntos de diretrizes a fim de auxiliar os desenvolvedores a criar conteúdo Web acessível. Assim, seguindo esses conjuntos, os desenvolvedores aumentam as chances de que o conteúdo Web criado estará disponível a todos os usuários.

Esses conjuntos de diretrizes são utilizadas no contexto de Interação HumanoComputador (IHC) e consistem não só de orientações para a solução de problemas conhecidos, com reconhecida eficácia, mas também de princípios, que são afirmações

MARTINS, G. A. A.; MOREIRA, D. A.; FORTES, R. P. M.

SEDA: Sistema para Exploração de Diretrizes de Acessibilidade

iSys - Revista Brasileira de Sistemas de Informação, Rio de Janeiro, Vol. 7, No. 4, p. 4-22, 2014. 
genéricas fundamentadas em pesquisas sobre como as pessoas aprendem e trabalham [Monk et al. 1993]. Dentre esses conjuntos, podemos destacar a WCAG e a Section 508.

As diretrizes criadas pelo W3C (World Wide Web Consortium), como um resultado dos trabalhos desenvolvidos pelo grupo WAI (Web Accessibility Initiative), são denominadas WCAG e são as que possuem um maior destaque na prática e estudos teóricos na área [Lazar et al. 2010]. As diretrizes propostas na WCAG visam tornar o conteúdo Web acessível a uma gama maior de pessoas com deficiência [W3C 2013].

A Section 508, proposta pelo HHS (Health \& Human Services), é uma emenda à Lei de Reabilitação da Força de Trabalho criada pelo governo federal dos Estados Unidos, que institui uma legislação sobre acessibilidade voltada para a tecnologia da informação e eletrônica, com a intenção de integrar pessoas com deficiência nos setores da tecnologia da informação [HHS 2013].

Ambos os conjuntos de diretrizes visam atender pessoas com deficiências como cegueira, baixa visão, surdez, baixa audição, dificuldades de aprendizagem, limitações cognitivas, limitações motoras, dificuldades na fala, fotossensibilidade e suas combinações [W3C 2013], sendo que a Section 508 estabelece normas de acessibilidade que devem ser atendidas quando se desenvolve um produto de software, enquanto a WCAG estabelece princípios a serem seguidos pelo desenvolvedor Web, pois possui suas recomendações voltadas para tornar aplicações Web acessíveis.

Esforços têm sido aplicados também para a utilização de ontologias na área de acessibilidade. Segundo Gruber [1993], o termo ontologia é definido como uma especificação formal e explícita de uma conceptualização compartilhada de um domínio de interesse:

- Conceituação se refere a um modelo abstrato de algum fenômeno no mundo, identificando os conceitos relevantes daquele fenômeno;

- Explícita significa que os conceitos utilizados e as restrições sobre seu uso são explicitamente definidos;

- Formal refere-se ao fato de que a ontologia deve ser legível pelas máquinas;

- Compartilhada refere-se à noção de que uma ontologia captura o conhecimento consensual, isto é, não é privado de algum indivíduo, mas aceito por um grupo.

Ou seja, uma ontologia deve ser um modelo de conhecimento compartilhado e consensual, acordado por uma comunidade [Devedzic, 2004], nesse caso, a que estuda acessibilidade. Ontologias são especificadas por meio de seus componentes básicos que são as classes, as relações, os axiomas e as instâncias, e são expressas por meio de uma linguagem padrão de construção [Almeida et al., 2003].

As ontologias são amplamente utilizadas para diferentes fins e em diferentes comunidades. Os esforços de utilização na área de acessibilidade consistem na facilitação da navegação Web para usuários que possuem alguma deficiência, a partir da definição de ontologias para classificar, de forma automática ou não, os elementos de uma página [Kouroupetroglou et al. 2006] [Ruslan et al. 2010]. Outro exemplo de utilização de ontologias é o apresentado por Tavares [Tavares et al. 2011], no qual ontologias são utilizadas para a classificação do acompanhamento realizado com usuários que possuem alguma deficiência, possibilitando a extração de conhecimento a partir do cruzamento dos dados dos pacientes. 
Para avaliar a potencial facilidade do sistema SEDA, como suporte à realização de classificações de diretrizes de acessibilidade em relação a deficiências, inicialmente foi desenvolvido um protótipo com base na arquitetura descrita próxima seção, e em seguida, foi definido e conduzido um estudo de caso. Nesse estudo de caso, as diretrizes de acessibilidade utilizadas foram as do conjunto de diretrizes do WCAG 2.0 e algumas das deficiências especificadas pela OMS.

\section{A arquitetura do sistema SEDA}

O sistema SEDA busca facilitar a exploração de diversos conjuntos de diretrizes de acessibilidade, utilizando como base um conjunto de descrições de deficiências. A facilidade de aprendizado e a eficiência de uso são características fundamentais para o sistema atingir seu propósito. Portanto, o protótipo foi construído utilizando apenas tecnologias Web, visando torna-lo portável e flexível uma vez que é executado em um navegador Web e não necessita de acesso à Internet, uma vez feito o download da aplicação. A Figura 1 ilustra a tela que exibe as informações que são exploradas no SEDA; a partir das listas de deficiências (à esquerda) e de diretrizes (à direita), os usuários podem interagir "associando-as", ao arrastá-las e juntá-las nas regiões centrais da tela.

\begin{tabular}{|c|c|c|c|c|c|c|}
\hline \multicolumn{7}{|c|}{ System for Exploration of Accessibilities Guidelines } \\
\hline \multirow{11}{*}{$\begin{array}{l}\quad \text { List of Desabilities } \\
\text { Attention functions } \\
\text { Memory functions } \\
\text { Psychomotor functions } \\
\text { Emotional functions } \\
\text { Perceptual functions } \\
\text { Thought functions } \\
\text { Higher-level cognitive functions } \\
\text { Mental functions of language } \\
\text { Calculation functions } \\
\text { Mental function of sequencing complex } \\
\text { movements } \\
\text { Experience of self and time functions } \\
\text { Functions of structures adjoining the eye } \\
\text { Sensations associated with the eye and } \\
\text { List of Used Desabilities } \\
\text { Intellectual functions }\end{array}$} & \multicolumn{3}{|c|}{ Drop Desabilities Here! } & \multicolumn{2}{|l|}{ Drop Guidelines Here! } & \multirow{2}{*}{$\begin{array}{l}\text { List of Guidelines } \\
\text { Audio-only and Video-only } \\
\text { (Prerecorded) }\end{array}$} \\
\hline & \multicolumn{3}{|c|}{ Seeing functions } & \multicolumn{2}{|l|}{ Non-text Content } & \\
\hline & \multirow{5}{*}{\multicolumn{3}{|c|}{$\begin{array}{l}\text { Sensory functions relating to sensing the presence of light and } \\
\text { sensing the form, size, shape and colour of the visual stimuli. } \\
\text { Inclusions: visual acuity functions; visual field functions; quality } \\
\text { of vision; functions of sensing light and colour, visual acuity of } \\
\text { distant and near vision, monocular and binocular vision; visual } \\
\text { picture quality; impairments such as myopia, hypermetropia, }\end{array}$}} & \multirow{2}{*}{\multicolumn{2}{|c|}{$\begin{array}{l}\text { All non-text content that is presented to the user has a text } \\
\text { alternative that serves the equivalent purpose }\end{array}$}} & \multirow{9}{*}{$\begin{array}{l}\text { Captions (Prerecorded) } \\
\text { Audio Description or Media Alternative } \\
\text { (Prerecorded) } \\
\text { Captions (Live) } \\
\text { Audio Description (Prerecorded) } \\
\text { Sign Language (Prerecorded) } \\
\text { Extended Audio Description } \\
\text { (Prerecorded) } \\
\text { Media Alternative (Prerecorded) } \\
\text { Audio-only (Live) } \\
\text { Info and Relationships } \\
\text { Sensorv Characteristics } \\
\text { List of Used Guidelines } \\
\text { Meaninfoul Sequence }\end{array}$} \\
\hline & & & & & & \\
\hline & & & & & & \\
\hline & & & & & & \\
\hline & & & & v & & \\
\hline & \multirow{2}{*}{\multicolumn{5}{|c|}{ Write your Rationale }} & \\
\hline & & & & & & \\
\hline & \multicolumn{5}{|c|}{$\begin{array}{l}\text { A descriçăo textual está diretamente } 1 \text { igada a funcionalidade dos leitores de conteúdo para pessoas } \\
\text { com deficiência visual. ok agora vamos para a quarta ligação.| }\end{array}$} & \\
\hline & \multicolumn{5}{|c|}{ Commit } & \\
\hline \multirow{2}{*}{ Intellectual functions } & Disability & Guideline & & Rationale & Rollback & \\
\hline & $\begin{array}{l}\text { Intellectual } \\
\text { functions }\end{array}$ & $\begin{array}{l}\text { Meaningful } \\
\text { Sequence }\end{array}$ & $\begin{array}{l}\text { Posso considerar que defi } \\
\text { sequência lógica. }\end{array}$ & ciências cognitivas podem prejudicar a percepção de & $\times$ & \\
\hline
\end{tabular}

Figura 1. Um exemplo de execução do sistema SEDA

Visando manter a flexibilidade, o sistema SEDA permite que o próprio usuário forneça as diretrizes de acessibilidade de sua escolha e as compare com um subconjunto de deficiências que pode ser selecionado dentro da própria interface. Essas diretrizes devem ser fornecidas para o sistema num formato padrão de entrada (JSON).

O formato padrão de entrada foi definido como sendo um arquivo JSON, devido a sua característica minimalista e de fácil manuseio. Como podemos ver na Figura 2, o primeiro campo representa uma chave única (id) utilizada pelo sistema para identificar aquele elemento, ficando a critério do usuário como ela será definida, exigindo apenas que ela seja única. 
$\mathrm{Na}$ sequência, tem-se os campos referentes ao título (title) da diretriz de acessibilidade e um texto complementar (complement) que auxilia o usuário, com informações adicionais para a adequada compreensão do elemento.

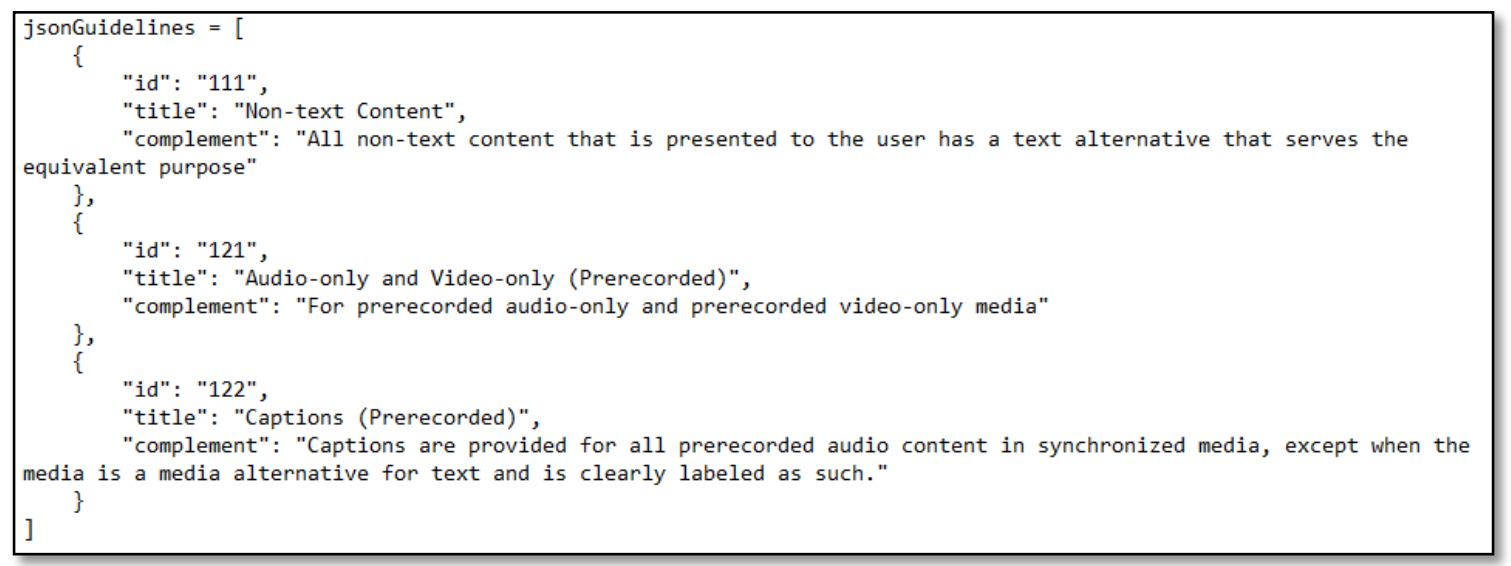

\section{Figura 2. Exemplo do JSON referente às diretrizes de acessibilidade}

Quanto ao conjunto das deficiências, foi utilizado um conjunto retirado da ontologia CIF, criada pela OMS [OMS 2013]. Desse modo, o SEDA se baseia em um conjunto de termos consolidados, padronizados e amplamente utilizados por especialistas da área da saúde, garantindo assim, credibilidade para os trabalhos realizados por meio desta ferramenta.

Essa ontologia CIF, ilustrada na Figura 3, é constituída de classes de deficiências que possuem subconjuntos contendo deficiências mais específicas, as quais podem possuir outros subconjuntos ou não.

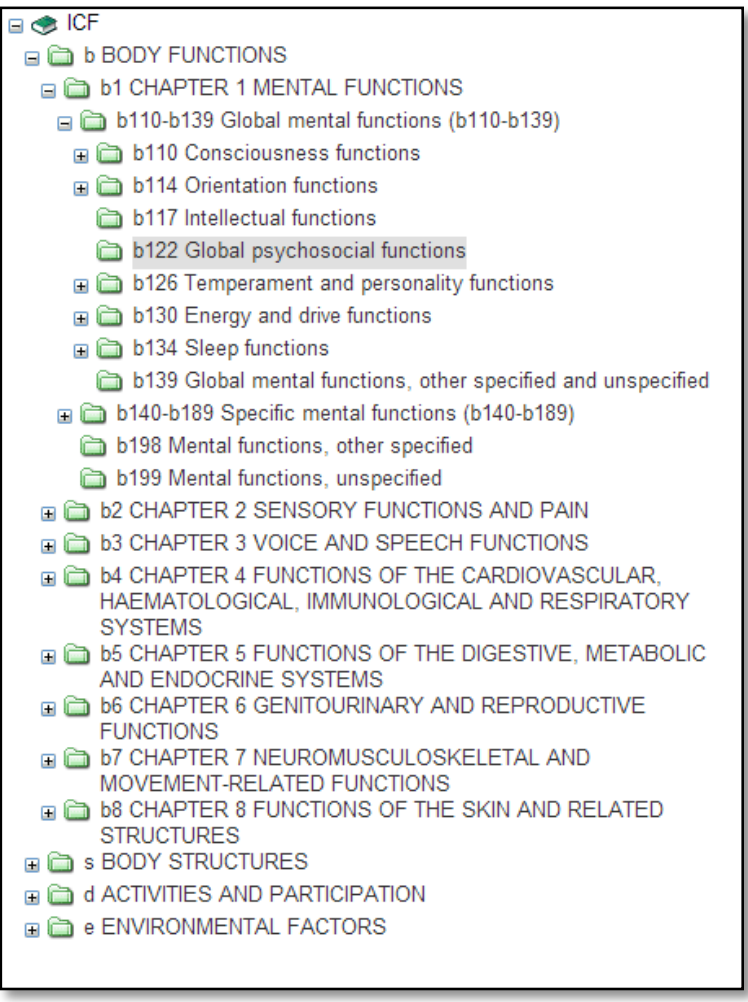

Figura 3. Exemplo de deficiências retiradas da ontologia CIF. 
Observa-se que, simplesmente exibir todas as informações presentes na ontologia CIF, levaria a uma visualização complexa dos elementos na interface, devido à grande quantidade de informação, Isso também acarretaria na criação de um conjunto de funcionalidades para o sistema, somente para o gerenciamento dessa grande quantidade de dados.

A utilização de todas as informações da ontologia CIF é necessária quando a mesma é utilizada num domínio clínico, pois é preciso diagnosticar exatamente qual é a deficiência que o paciente possui. Todavia, no contexto da classificação de diretrizes de acessibilidade, que normalmente trata as deficiências com o objetivo de descrever o problema para identificar as barreiras de acessibilidade na Web, a presença de dados muitos específicos poderia dificultar muito a classificação a ser realizada pelo usuário.

Assim, foi decidido se limitar a quantidade de dados retirados da ontologia. Portanto, são utilizadas as descrições das deficiências detalhadas em até quatro níveis de profundidade, a partir da raiz da ontologia. Essa limitação foi estabelecida após realizarmos uma análise aprofundada dos dados a serem utilizados, e constatarmos que, das 116 deficiências utilizadas, 57 deficiências tinham apenas quatro níveis de detalhamento possíveis. Para as 59 deficiências restantes, optamos por não utilizar os níveis subsequentes, pois notamos que, em muitos casos, as informações eram as mesmas com pequenas alterações, o que acarretaria em uma grande quantidade de dados de entrada muito similares o que comprometeria a simplicidade proposta no sistema.

Depois das informações sobre as diretrizes de acessibilidade (Figura 2) terem sido carregadas do seu respectivo arquivo JSON (a ontologia CIF faz parte do sistema e é sempre carregada), elas são armazenadas no navegador Web por meio do webstorage, presente no HTML5. Na Figura 4 encontra-se ilustrada a arquitetura dos componentes do sistema SEDA.

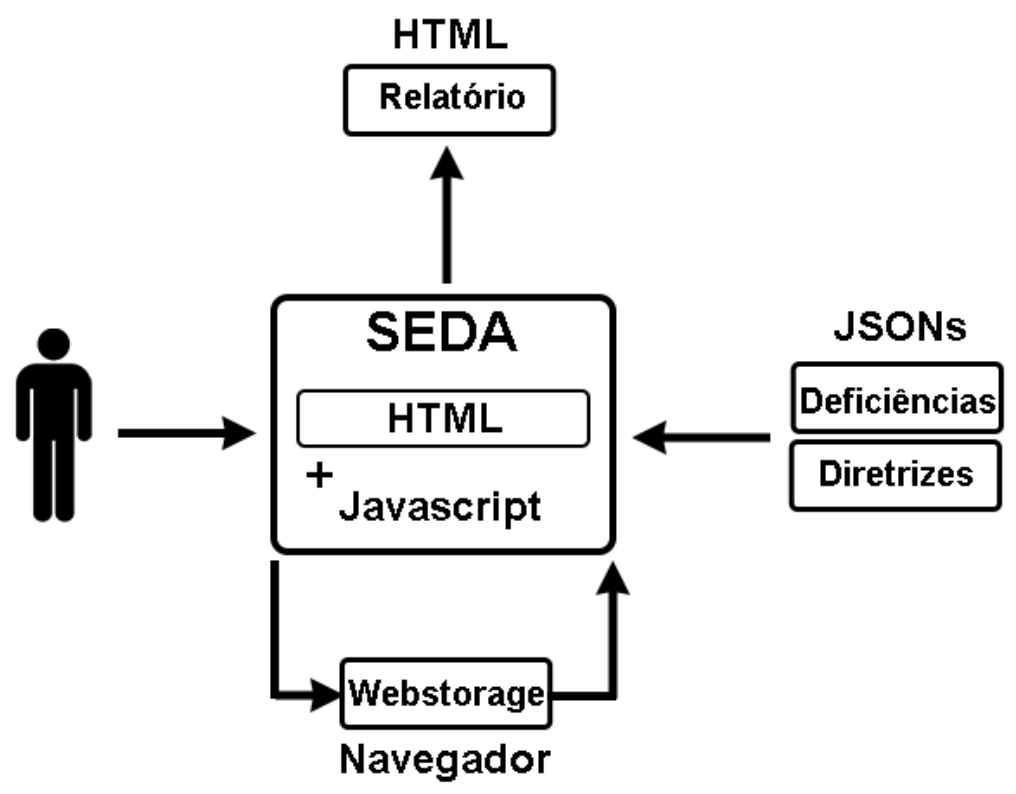

Figura 4. Arquitetura dos componentes do sistema SEDA. 
Com o webstorage, podemos armazenar grandes quantidades de dados no próprio navegador do usuário (por meio de funções Javascript), o que permite ao usuário, que esteja trabalhando e queira parar por algumas horas (ou até mesmo dias), continuar o trabalho posteriormente, sem a exigência de manter uma conexão com a Internet ou de criar um banco de dados dedicado no servidor. As informações armazenadas, por meio do webstorage, ficam salvas em pares chave/valor por tempo indeterminado e podem ser acessadas apenas pela página que criou as informações e sem comprometimento do desempenho do navegador.

Após a formatação dos dados de entrada, eles são apresentados nas regiões laterais da tela do sistema no formato de listas, nas quais o usuário pode navegar entre os dados e selecioná-los por meio da funcionalidade Drag and Drop (ou arrastar-esoltar), presente no conjunto de funcionalidades HTML5. A opção por interação com estilo arrastar-e-soltar foi escolhida para viabilizar uma maneira mais eficiente de manipular um grande volume de informação, relacionando-as por meio da interação por movimento de aproximação dos itens relacionados. A facilidade propiciada com essa maneira de interagir com os itens de informação apresentados, arrastando-os para trazelos próximos no centro da tela, imita a metáfora de aproximação de objetos físicos, e sua realização por meio de gestos em tela touchscreen, em geral, é apreciada pela maioria dos usuários, que os encaram como interação mais natural. Observamos que, de fato, trata-se de uma potencial barreira para usuários cegos ou que possuam limitação motora, porém, esta primeira versão do sistema SEDA é um protótipo e motiva outras investigações futuras, no sentido de ampliar efetivamente o alcance para todos.

Na Figura 5, podemos ver duas listas que apresentam deficiências a serem utilizados no sistema SEDA. Uma contendo os dados ainda não utilizados e outra contendo os dados que já o foram pelo menos uma vez.

$\quad$ List of Desabilities
Psychomotor functions
Emotional functions
Perceptual functions
Thought functions
Mental functions of language
Mental function of sequencing complex
movements
Functions of structures adjoining the eye
Sensations associated with the eye and
adjoining structures
Hearing functions
Vestibular functions
Sensations associated with hearing and
vestibular function
List of Used Desabilities
Intellectual functions
Seeing functions
Attention functions
Memory functions
Calculation functions
Experience of self and time functions
Higher-level cognitive functions

Figura 5. Exemplo de utilização das listas de Deficiências

Essa visualização em listas permite que, não só o usuário possa relacionar uma deficiência com mais do que uma diretriz e vice-versa, mas também possa obter uma 
rápida visualização das deficiências utilizadas até o momento, garantindo assim o princípio proposto para a aplicação, de facilitar a organização das informações.

Quando ambos os itens de informação, referentes a diretriz de acessibilidade e a deficiência, são selecionados para representar uma relação, além da apresentação de textos explicativos para facilitar sua compreensão, é iniciado o processo de justificativa para a relação definida. O processo de justificativa nada mais é do que o registro do motivo pelo qual o usuário estabeleceu aquela relação; para tanto, ao usuário é exibida uma caixa de texto (Write your Rationale, na Figura 1). Dessa forma, ao término do processo de classificação das diretrizes, teremos o registro de porquê aquelas relações foram decididas daquela maneira. Esse registro é importante uma vez que ajuda o usuário a se lembrar de porquê relacionou os elementos dessa forma e reforça o trabalho realizado pelo usuário.

A apresentação dos relacionamentos criados pelo usuário é realizada na parte inferior da tela por meio de uma tabela (Commit, na Figura 1). Nela, além de visualizar todas as operações realizadas até o momento, é possível também remover relações criadas anteriormente, caso seja detectado um erro pelo usuário. Assim, os dados voltam para as suas posições originais e um novo relacionamento pode ser criado com os dados utilizados. Esse é um mecanismo para correção de erros que é simples de ser utilizado.

Por fim, temos a geração de um relatório contendo informações sobre os dados utilizados. Nesse relatório, no formato HTML, é criada uma tabela contendo todas as relações definidas pelo usuário, juntamente com suas respectivas justificativas e também, listas contendo o número de vezes que cada elemento foi utilizado. Dessa forma, o usuário consegue visualizar todas as informações num só lugar e consegue, a partir delas, obter conhecimentos que serão úteis para a continuidade do seu trabalho.

\section{Método de Pesquisa}

Um estudo de caso foi realizado para avaliar o protótipo do sistema SEDA. Ele visou avaliar a execução do protótipo por usuários que se interessassem por explorar as diretrizes de acessibilidade, identificando para quais deficiências elas seriam mais adequadas. Portanto, para a condução desse estudo foi necessária a participação de indivíduos que possuíssem ao menos algum conhecimento sobre diretrizes de acessibilidade.

Para tanto, definimos três questões principais de verificação durante o uso do sistema, para posterior análise:

(1) O sistema irá realmente auxiliar os pesquisadores a explorarem as diretrizes de acessibilidade ajudando-os a identificar para quais deficiências elas seriam mais adequadas?

(2) Quais as possíveis falhas no sistema que podem ser corrigidas ou possíveis funcionalidades que podem ser melhoradas?

(3) Quais as características do sistema que se destacaram no auxílio à exploração das diretrizes de acessibilidade?

Para encontramos a respostas para essas questões, a coleta de dados foi realizada de duas maneiras distintas. De acordo com a definição de [Lethbridge et al. 2005], podemos separar as técnicas de coleta de dados em três níveis: primeiro, quando o

MARTINS, G. A. A.; MOREIRA, D. A.; FORTES, R. P. M. 
pesquisador está em contato direto com os sujeitos e os dados são coletados em tempo real. Segundo nível, quando o pesquisador indiretamente coleta dados sem interagir diretamente com os sujeitos. E terceiro nível, quando o pesquisador se utiliza da análise independente de artefatos ou documentos já existentes.

Nesse estudo, foram utilizadas para coleta de dados, duas técnicas de primeiro nível: entrevistas presenciais e observação do sujeito ao utilizar o sistema para realizar um conjunto de tarefas pré-estabelecidas.

As entrevistas presenciais foram realizadas em duas sessões. A primeira, realizada antes da observação de uso do sistema, para coletar informações sobre o participante e sua experiência com diretrizes de acessibilidade e suas deficiências, e a segunda entrevista, realizada posteriormente ao uso do sistema SEDA, para obter informações sobre a experiência do usuário durante a utilização. Ambas as entrevistas ocorreram de maneira semiestruturada, ou seja, a ordem das questões não segue necessariamente uma maneira linear. Essa abordagem possibilita ao entrevistador incluir ou remover perguntas de acordo com o rumo da entrevista, tornando-a assim uma entrevista oportunista, o que é bastante útil para se aumentar ou aprofundar o entendimento [Lazar et al. 2010].

Para a coleta de dados durante a observação, foi utilizado um computador instrumentado para a gravação do áudio da interação do usuário com o sistema. Assim, o usuário recebe uma serie de tarefas pré-estabelecidas para que as execute por meio do sistema e nos diga, em voz alta (para a gravação do áudio), o que está ocorrendo e quais são seus pensamentos durante a execução das tarefas. Essa técnica tem o nome de think aloud. Também nesta etapa da coleta de dados, com o objetivo de coletar dados quantitativos, foram extraídas as seguintes métricas: falhas no sistema por tarefa, onde é medido o número de falhas de usabilidade no sistema encontrados durante a realização das tarefas pelo usuário; e tempo por tarefa, onde é medido o tempo que o usuário levou para realizar cada uma das tarefas. Ambas as métricas foram estipuladas para verificar as questões citadas anteriormente nesse estudo de caso.

A partir dos dados coletados, foi realizado o processo de triangulação, onde se aborda o objeto de estudo por diferentes ângulos. Essa técnica é fundamental quando se está realizando um estudo qualitativo, pois o material coletado em geral é muito rico e extenso, porém menos preciso do que dados quantitativos [Runeson and Höst 2008].

Logo, de acordo com [Stake 1995], existem quatro tipos de triangulação que podem ser realizadas num estudo de caso: triangulação de dados, triangulação de observadores, triangulação metodológica e triangulação teórica. Neste trabalho foram realizados três tipos de triangulação:

- Triangulação metodológica: com o objetivo de validar e aumentar a confiabilidade dos dados coletados, serão combinadas uma estratégia qualitativa (entrevista) com uma estratégia quantitativa (métricas na observação). Embora essa abordagem quantitativa não seja suficiente para que conclusões sejam estatisticamente válidas, elas irão colaborar para a validade dos dados coletados, seja sustentando os dados das entrevistas ou expondo possíveis divergências.

- Triangulação de dados: do mesmo modo que na triangulação metodológica, a triangulação de dados se dará pela coleta de dados de duas fontes diferentes, nas entrevistas, de maneira direta, e através de gravações de áudio, de maneira indireta. 
O uso de fontes de dados distintas provê oportunidades para se obter insights que poderiam ser mais difíceis de se obter quando utilizando-se apenas uma forma de coleta de informações [Lazar et al. 2010].

- Triangulação de observador: durante a fase de análise dos dados, todo o material coletado durante as entrevistas e observação do uso da ferramenta serão analisados pelo autor com o auxílio de um segundo pesquisador. Dessa forma, busca-se evitar que as análises sejam influenciadas pelas opiniões pessoais dos pesquisadores e reflitam de maneira mais fiel os fatos.

Com o uso dessas triangulações citadas e analisando os dados das três etapas de maneira cruzada, foram obtidos os resultados apresentados a seguir.

\section{O Estudo de Caso}

O estudo foi conduzido com dois indivíduos cujos nomes são Fernando e Felipe, nomes fictícios a fim de manter a confidencialidade dos participantes. Os participantes assinaram o Termo de Consentimento Livre e Esclarecido (TCLE) e assim puderam realizar o Estudo de Caso, conforme as orientações legais. A partir da pré-entrevista foi possível conhecer um pouco mais sobre os participantes.

Fernando possui 26 anos, e está atualmente realizando um projeto de mestrado, na Universidade de São Paulo, relacionado com acessibilidade e usabilidade em aplicações Web. Ele trabalha com diretrizes de acessibilidade e deficiências há cerca de dois anos e meio, e, atualmente, está realizando um processo de classificação das diretrizes de acessibilidade com suas respectivas deficiências, a fim de auxiliar desenvolvedores de aplicativos Web. Apesar de não ter tido contato, durante seu trabalho, com nenhum grupo de usuários com deficiências específicas, como cegos ou idosos, participou no desenvolvimento de vários projetos e auxilia um grande número de desenvolvedores.

Já Felipe, possui 22 anos e também está realizando mestrado na Universidade de São Paulo. Sua área de especialidade é a acessibilidade para dispositivos móveis e ele trabalha com diretrizes de acessibilidade há cerca de um ano. Felipe possui uma visão global das deficiências, não tendo contato nenhum com grupos de usuários com deficiências específicas. Ele entretanto possui experiência em avaliações de usabilidade e acessibilidade, o que pode vir a auxiliar na definição de novos requisitos para trabalhos futuros.

Durante a fase de observação, esses dois usuários completaram um conjunto de quatro tarefas pré-estabelecidas. Foram gravados 23:55 minutos de áudio, na sessão do Fernando, e 31:36 minutos de áudio, na sessão do Felipe. Para propósito de comparação, o tempo utilizado em cada uma das quatro tarefas pode ser visto no gráfico da Figura 6.

MARTINS, G. A. A.; MOREIRA, D. A.; FORTES, R. P. M.

SEDA: Sistema para Exploração de Diretrizes de Acessibilidade

iSys - Revista Brasileira de Sistemas de Informação, Rio de Janeiro, Vol. 7, No. 4, p. 4-22, 2014. 


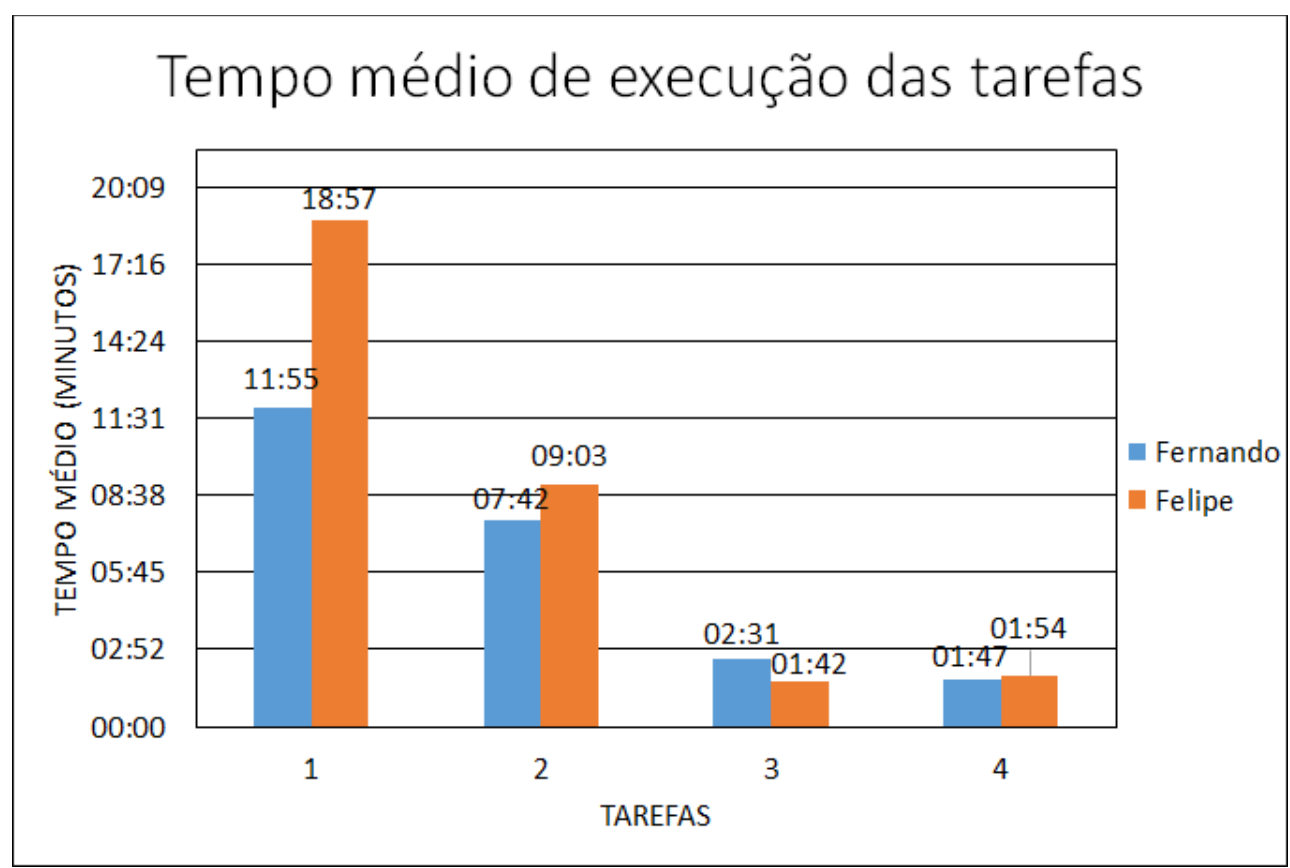

Figura 6. Tempo utilizado pelos usuários em cada uma das tarefas.

A tarefa 1 consistia em relacionar cinco deficiências com cinco diretrizes de acessibilidade, apresentando uma justificativa para cada uma delas. Como já possui alguma experiência com classificação de diretrizes, Fernando, de modo geral, não encontrou dificuldades para a realização dessa tarefa. Apesar de encontrar alguns problemas de usabilidade simples, como o modo como as descrições são exibidas para o usuário e o tamanho da caixa de seleção para executar a função drag and drop, essas dificuldades não interferiram na execução da tarefa.

Já Felipe, não conseguiu desempenhar a tarefa com a mesma maestria que Fernando. Isso ocorreu, devido a dificuldades de compreensão do conteúdo apresentado, uma vez que a ferramenta é apresentada em inglês, e também ao fato de Felipe não estar totalmente familiarizado com as deficiências apresentadas. Todavia, isso não impediu que o mesmo completasse a tarefa proposta e apontasse questões de usabilidade interessantes para o projeto do sistema, como: sugestão de uma localização melhor para os botões, observação da ausência de ao menos um campo de busca na ferramenta e proposta de outras formas para realizar a seleção dos elementos na aplicação.

A tarefa 2 consistia em realizar relacionamentos de um para muitos, logo é necessário vincular uma das deficiências utilizadas anteriormente com uma nova diretriz de acessibilidade e vice-versa. Para realizar essa tarefa, Fernando teve dificuldades para encontrar os elementos que ele havia usado na tarefa anterior. Isso ocorreu devido aos elementos presentes nas listas de deficiências e diretrizes estarem ordenados por elementos semelhantes, o que de certa forma dificulta a pesquisa por um elemento específico. Outro problema observado foi a apresentação dos elementos já selecionados apenas nas listas de elementos usados, sendo mais interessante apresentar esses elementos em ambas as listas, com o intuito de facilitar a busca por elementos específicos. Com relação a Felipe, apesar de também ter apontado tais problemas, eles não causaram dificuldades para a resolução da tarefa.

Como esperado, as tarefas 3 e 4 foram os que levaram menor tempo para serem completadas, pois elas são menos complexas e, portanto, a probabilidade de o sujeito 
passar por dificuldades para a realização das tarefas seria menor. Na tarefa 3 , o usuário deveria realizar um cenário de correção de erros, ou seja, deveria remover um relacionamento criado, por meio da funcionalidade rollback do sistema, e vincular novamente elementos com uma nova justificativa.

Já na tarefa 4, o usuário deveria gerar um relatório, com os dados utilizados nas tarefas anteriores, e, com base nele, dizer ao pesquisador qual foi o número de vezes que cada diretriz de acessibilidade e cada deficiência foram utilizadas. Essa tarefa foi concebida com o intuito de avaliar se o protótipo do relatório estava conciso e se possibilitava ao usuário achar as informações, que ele estivesse procurando, de forma eficiente.

Uma observação importante em relação à tarefa 3 é o fato de que Felipe notou que, ao realizar a funcionalidade de rollback do sistema, a mesma não pergunta ao usuário se ele realmente quer realizar essa operação. Isso pode acarretar um grande problema, caso o usuário selecione essa funcionalidade por engano e remova relacionamentos que, a princípio, estavam corretos. Esse problema representa uma séria questão crítica de usabilidade.

O gráfico da Figura 7 mostra o número de problemas de usabilidade encontrados no sistema, por ambos os usuários, durante a execução de cada uma das tarefas. Uma vez que as tarefas 1 e $2 \mathrm{~s}$ são as que envolvem mais interação, foram também as que apresentaram também o maior número de problemas de usabilidade. Levando-se em consideração que, uma das razões para a execução desse estudo de caso era encontrar problemas de usabilidade, podemos afirmar que a execução do mesmo foi de grande valia para o contínuo aprimoramento do sistema SEDA.

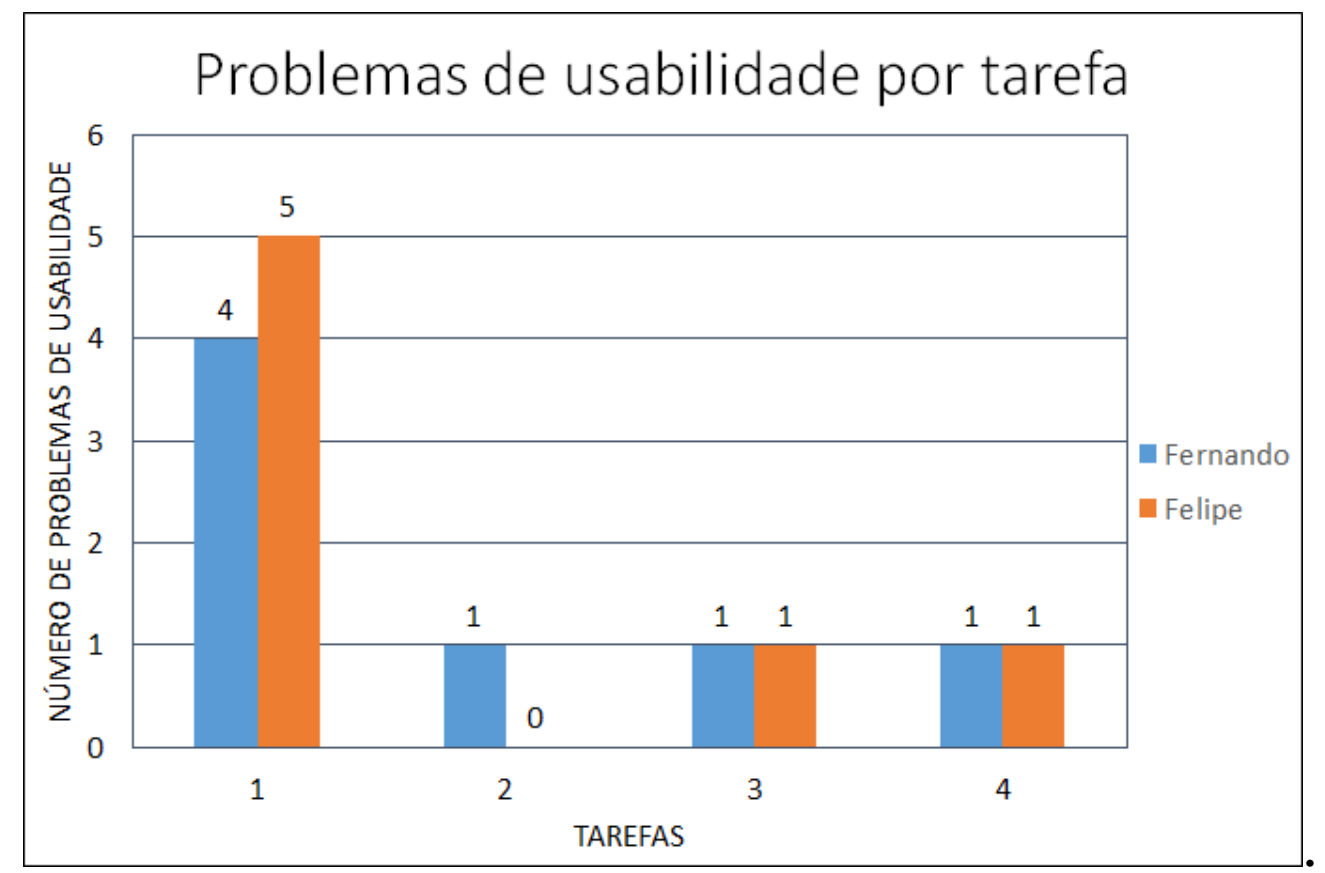

Figura 7. Número de falhas de usabilidade encontradas no sistema por ambos os usuários durante a execução de cada uma das tarefas. 


\section{Discussão}

O Estudo de caso realizado com dois usuários para a avaliação do sistema SEDA possibilitou a identificação de problemas de usabilidade. Além disso, foram obtidas, a partir da fase de observação das tarefas e a partir das entrevistas realizadas, informações que destacaram a utilidade do sistema e também apresentaram novas possíveis aplicações para o projeto proposto. A seleção dos usuários foi realizada levando em consideração o conhecimento necessário para a utilização da ferramenta, bem como a contribuição que a avaliação realizada por tais usuários poderia ser utilizada para a melhoria do protótipo, vide suas experiências na área (o que reduziu bastante o universo de possíveis voluntários).

A quantidade de usuários, para realização do estudo, vem de encontro com a necessidade atual do projeto, cuja prioridade foi identificar a maior quantidade de informações a respeito da usabilidade da ferramenta, com o intuito de otimizar sua utilização. Logo, tendo em vista a limitação do número de usuários no Estudo de Caso, e o fato de que a quantidade de problemas encontrados (durante a avaliação com os usuários) não aumenta proporcionalmente com a quantidade de usuários selecionados [Nielsen and Landauer 1993], dois usuários foi um número adequado para a realização desse estudo de caso.

Uma conclusão inicial, a partir do estudo de caso, é que o sistema SEDA auxilia o pesquisador na exploração de diretrizes de acessibilidade, o qual foi o principal objetivo do sistema. Tal conclusão é apoiada pelo testemunho fornecido por Fernando, que, durante a última fase de entrevistas, nos disse que o sistema é prático e simples devido ao fato de usar uma distribuição da informação respeitando o formato: de deficiências no lado esquerdo e de diretrizes de acessibilidade no lado direito da interface. Segundo ele, isso possibilita encararmos o sistema como um desafio a ser alcançado, como se fosse um jogo de ligar elementos como os que normalmente encontramos em literatura infantil.

Outro ponto interessante, destacado por Fernando, presente no sistema SEDA é a necessidade de se justificar cada relacionamento criado. Segundo ele, normalmente durante o processo de relacionamento de diretrizes de acessibilidade a deficiências, nenhuma justificativa é exigida, o que acaba deixando lacunas para possíveis erros de relacionamento. Logo, ao realizar o processo de justificativa, o pesquisador acaba refletindo melhor sobre o porquê de estar realizando aquele relacionamento, o que diminui a chance de se criar um relacionamento não fundamentado.

Além disso, ambos os usuários relataram, durante as entrevistas finais, que as deficiências apresentadas pelo sistema SEDA (na lista à esquerda) não estavam uniformemente selecionadas, o sistema às vezes mostrava deficiências genéricas demais ou específicas demais. Tal fato se deve ao critério de se utilizar apenas quatro níveis de profundidade, em relação à raiz da ontologia CIF, para se limitar a utilização das deficiências presentes nessa ontologia. Portanto, podemos concluir que a ontologia CIF não é simétrica o bastante para se utilizar esse tipo de delimitação, sendo necessário o auxílio de um especialista da área da saúde, para nos auxiliar com a escolha acertada das deficiências que devem ser apresentadas no sistema.

Por fim, a partir das informações coletadas nas entrevistas, pôde-se observar que o sistema SEDA pode ser utilizado, não só por pesquisadores que buscam explorar 
diretrizes de acessibilidade, mas também por desenvolvedores que queiram focar seu trabalho num grupo de usuários com deficiências específicas, como cegos ou surdos, por exemplo. O sistema SEDA possibilita claramente verificar a relação das diretrizes com determinadas deficiências, proporcionando uma visão especializada que pode ser explorada nas técnicas de boas práticas de desenvolvimento. Normalmente, desenvolvedores não têm acesso a esse tipo de informação específica, como aquelas verificadas na lista de deficiências, o que limita a garantia de acessibilidade do software desenvolvido a apenas alguns grupos com deficiências específicas. No entanto, quando se depara com as diversas possibilidades encontradas por outros tipos de deficientes, o desenvolvedor em geral as desconhece e o auxílio de SEDA pode lhe amparar neste quesito.

\section{Conclusões e Trabalhos Futuros}

Este artigo descreveu o protótipo do sistema SEDA, um sistema desenvolvido para apoiar a exploração de diretrizes de acessibilidade, auxiliando os usuários tanto na identificação de deficiências, para as quais já foram criadas diretrizes de acessibilidade, quanto para identificar os limites existentes ao realizar uma comparação entre diretrizes de acessibilidade e deficiências.

A proposta do sistema SEDA foi de viabilizar uma interação mais ágil para manipulação dos itens de informação a serem associados, os quais estão disponíveis em diferentes fontes de classificação. No caso do SEDA, foram disponibilizadas as diretrizes WCAG confrontando-se com as possíveis deficiências descritas pelo CIF. De fato, o volume de informações dessas fontes é extenso e exige que o foco de atenção durante as interações seja dedicado; o modo de interação de arrastar-e-soltar foi então implementado viabilizando que os pesquisadores tivessem melhor eficiência na sua principal tarefa.

Como trabalhos futuros, os autores pretendem investigar não só novas formas de apresentar as descrições para os elementos da interface, de forma mais clara, inclusive levando em consideração a possibilidade de internacionalizar o sistema, além de oferecer também novas formas de exibição e seleção dos elementos presentes nas listas de diretrizes de acessibilidade e deficiências. Dessa forma, pretendemos contribuir para melhorar as características principais do SEDA, na sua próxima versão, em termos de facilidade de aprendizado e eficiência de uso.

Outra pesquisa futura seria o desenvolvimento de um módulo, no SEDA, que permitisse a visualização dos relacionamentos criados por outros pesquisadores. Desse modo, seria possível ter acesso de forma simples e rápida a conjuntos de diretrizes e deficiências relacionadas pela comunidade, permitindo não só visualizar relacionamentos criados, mas também filtrar deficiências específicas através da seleção de diretrizes de acessibilidade, e vice-versa. Esta funcionalidade auxiliaria na questão da utilização da ferramenta por desenvolvedores, e também com o compartilhamento de relacionamentos criados entre pesquisadores.

Um importante trabalho a ser continuado, a partir desta proposta do sistema SEDA, consiste em sua validação. Desse modo, uma potencial inclusão do sistema SEDA a um ambiente de avaliação de acessibilidade, já desenvolvido, será considerada. O ambiente de avaliação é o A4U (Ambiente para Análise de Avaliações de 
Acessibilidade e Usabilidade na Web) [Amaral et al 2012]. O A4U permite incorporar resultados de avaliações de diferentes ferramentas semiautomáticas em um mesmo ambiente, relacionando seus apontamentos em forma de apoio a sua avaliação, que não consistirá apenas na correção de erros. A ferramenta instiga o desenvolvedor a pensar nos problemas, contribuindo para que as recomendações sejam consideradas previamente em futuros desenvolvimentos.

Investigar acessibilidade, sem ter a experiência de lidar com o público alvo, é um aspecto que deve ser perseguido por parte das pesquisas que serão realizadas também a partir do sistema SEDA. O sistema deve ser, portanto, avaliado por um número maior de usuários, incluindo pessoas com deficiências, para incrementar as garantias de acessibilidade e ampliar o potencial de uso e resultados do sistema. Sob a perspectiva experimental, o contato com esses usuários possibilita contribuições nesta área tão essencial [Freire et al 2013]. Mesmo que minimamente, é importante colaborar na experiência diária dessas pessoas, que são visivelmente prejudicadas pela falta de interesse e conhecimento das empresas para tornar seus conteúdos acessíveis.

Por fim, é interessante mencionar que, do ponto de vista técnico, o protótipo do sistema SEDA possui uma arquitetura bastante flexível, que permite que todas as alterações de usabilidade, propostas pelos usuários do estudo de caso, sejam realizadas sem afetar as funcionalidades já existentes.

\section{Agradecimentos}

Agradecemos ao ICMC-USP e ao CNPq, pelo suporte financeiro.

\section{Referências}

[Accessible 2013] Accessible Project (2013). Applications Design and Development. http://www.accessible-eu.org/

[Almeida et al. 2003] Almeida, M. B.; Bax, M. P. (2003) Uma visão geral sobre ontologias: pesquisa sobre definições, tipos, aplicações, métodos de avaliação e de construção. Ciência da Informação, Brasilia, v. 32, n. 3, p. 7-20

[Amaral et al 2012] Amaral, L. A.; Bittar, T. J. e Fortes, R. P. M. (2012) Um ambiente de análise para comparar resultados de avaliações de acessibilidade e usabilidade na Web. In: Conferência IADIS Ibero Americana WWW/INTERNET 2012, 2012, Madrid. Actas da Conferência Lisboa: IADIS, 2012. v. 01. p. 166-170.

[Brajnik 2013] Brajnik, G. (2013). Barrier Walkthrough. http://sole.dimi.uniud.it/ giorgio.brajnik/index.html

[Devedzic 2004] Vladan Devedzic. (2004). Education and the Semantic Web. Int. J. Artif. Intell. Ed. 14, 2 (April 2004), 165-191.

[Freire et al 2013] Freire, A. P.; Lara, S. M. A. de; Fortes, R. P. M. Avaliação da Acessibilidade de Websites por Usuários com Deficiência. In: José Maria N. David; Luciana C. de Castro Salgado. (Org.). Tutoriais do X Simpósio Brasileiro de Sistemas Colaborativos e XII Simpósio Brasileiro sobre Fatores Humanos em Sistemas Computacionais. 1.ed. Porto Alegre RS: SBC, 2013, v. 1, p. 31-64.

[Gruber 1993] Thomas R. Gruber. (1993). A translation approach to portable ontology specifications. Knowl. Acquis. 5, 2 (June 1993), 199-220. 
DOI=10.1006/knac.1993.1008 http://dx.doi.org/10.1006/knac.1993.1008

[HHS 2013] U.S. Department of Health \& Human Services (2013). Section 508 of the Rehabilitation Act. http://www.hhs.gov/accessibility.html

[IBGE 2010] Instituto Brasileiro de Geografia e Estatística. Census (2010). http://www.ibge.gov.br.

[Iwase and Murata 2002] Iwase, H., Murata, A. (2002). Empirical study on improvement of usability for touchpanel for elderly - comparison of usability between touch-panel and mouse. In: IEEE International Conference on Systems, Man and Cybernetics 2002, pp. 252-257. IEEE Computer Society, Los Alamitos (2002)

[Jin et al. 2007] Zhao Xia Jin, Tom Plocher, and Liana Kiff (2007). Touch screen user interfaces for older adults: button size and spacing. In Proceedings of the 4th international conference on Universal access in human computer interaction: coping with diversity (UAHCI'07), Constantine Stephanidis (Ed.). Springer-Verlag, Berlin, Heidelberg, 933-941.

[Kouroupetroglou et al. 2006] Kouroupetroglou, C., Salampasis, M. \& Manitsaris, A. (2006). 'A Semantic-web based framework for developing applications to improve accessibility in the WWW', in W4A: Proceedings of the 2006 international crossdisciplinary workshop on Web accessibility (W4A), ACM Press, pp. 98-108.

[Lazar et al. 2010] Lazar, J.; Feng, J.; Hochheiser, H. (2010). Research methods in human-computer interaction. Indianapolis. Wiley 2010.

[Lee et al. 2009] Ju-Hwan Lee, Ellen Poliakoff, and Charles Spence (2009). The Effect of Multimodal Feedback Presented via a Touch Screen on the Performance of Older Adults. In Proceedings of the 4th International Conference on Haptic and Audio Interaction Design (HAID '09), M. Ercan Altinsoy, Ute Jekosch, and Stephen Brewster (Eds.). Springer-Verlag, Berlin, Heidelberg, 128-135. DOI=10.1007/978-3642-04076-4_14 http://dx.doi.org/10.1007/978-3-642-04076-4_14

[Lethbridge et al. 2005] Lethbridge, T. C.; Sim, S. E.; Singer, J. (2005). Studying software engineers: Data collection techniques for software field studies. Empirical Softw. Engg. v. 10, n. 3, p. 311-341,2005. http://dx.doi.org/10.1007/s10664-005$1290-\mathrm{x}$

[Monk et al. 1993] Monk, A., Wright, P., Haber, J., and Davenport, L. (1993). Improving Your Human-Computer Interface: A Practical Technique. Prentice Hall International, Hemel Hempstead, U.K.

[Nielsen and Landauer 1993] Nielsen J.; Landauer T. K. (1993) A mathematical model of the finding of usability problems. In Proceedings of the INTERACT '93 and CHI '93 Conference on Human Factors in Computing Systems (CHI '93). ACM, New York, NY, USA, 206-213. DOI=10.1145/169059.169166 http://doi.acm.org/10.1145/169059.169166

[NU 2013] Nações Unidas (2013). Deficiências. http://www.un.org/disabilities.

[Protégé 2013] Protégé (2013). http: / / protege.stanford.edu /

MARTINS, G. A. A.; MOREIRA, D. A.; FORTES, R. P. M.

SEDA: Sistema para Exploração de Diretrizes de Acessibilidade

iSys - Revista Brasileira de Sistemas de Informação, Rio de Janeiro, Vol. 7, No. 4, p. 4-22, 2014. 
[Ocenasek 2009] Pavel Ocenasek. 2009. Universal Access to the Internet Web Pages for Users with Special Needs. In Proceedings of the 5th International Conference on Universal Access in Human-Computer Interaction. Part III: Applications and Services (UAHCI '09), Constantine Stephanidis (Ed.). Springer-Verlag, Berlin, Heidelberg, 764-767. DOI=10.1007/978-3-642-02713-0 81 http://dx.doi.org/10.1007/978-3-642-02713-0_81

[OMS 2013] Organização Mundial da Saúde (2013). Ontologia CIF. http://bioportal.bioontology.org/ontologies/ICF

[Runeson and Höst 2008] Runeson, P.; Höst, M. (2008). Guidelines for conducting and reporting case study research in software engineering. Empirical Software Engineering, v. 14, n. 2, p. 131-164, 2008. http://www.springerlink.com/index/10.1007/s10664-008-9102-8

[Ruslan et al. 2010] Ruslan R. Fayzrakhmanov, Max C. Göbel, Wolfgang Holzinger, Bernhard Krüpl, and Robert Baumgartner (2010). In Proceedings of the 19th International Conference on World Wide Web (WWW'2010), Raleigh, USA, April 26-30, 2010, pages 1087-1088, New York, 2010. ACM.

[Stake 1995] Stake, R. (1995). The art of case study research. Sage Publications Inc, http://books.google.com/books?hl=no\&lr=\&id=ApGdBx76b9kC\&oi=fnd\&pg=PR11 $\& d q=$ case + study\&ots $=$ KrNKk2Fnbo\&sig $=$ tBVSrcFVI4tzDG3AzErMhF_ xe4

[Tavares et al. 2011] Tavares, João; Barbosa, Jorge; Costa, Cristiano; Yamin, Adenauer; Real, Rodrigo (2011). "Hefestos: a Model for Ubiquitous Accessibility Support", 4th International Conference on Pervasive Technologies Related to Assistive Environments, PETRA'2011, Crete, Greece.

[Thatcher et al. 2003] Thatcher, J., Bohman, P., Burks, M., Henry, S. L., Regan, B., Swierenga, S., Urban, M. D., \& Waddell, C. D. (2003). Constructing accessible web sites. Birmingham, UK: Glasshaus, Ltd.

[Tsai and Lee 2009] Wang-Chin Tsai and Chang-Franw Lee (2009). A Study on the Icon Feedback Types of Small Touch Screen for the Elderly. In Proceedings of the 5th International on ConferenceUniversal Access in Human-Computer Interaction. Part II: Intelligent and Ubiquitous Interaction Environments (UAHCI '09), Constantine Stephanidis (Ed.). Springer-Verlag, Berlin, Heidelberg, 422-431. DOI=10.1007/978-3-642-02710-9_46 http://dx.doi.org/10.1007/978-3-642-02710946

[Watanabe et al. 2011] Watanabe, W. M.; Fortes, R. P. M., Pimentel, M. G. C. 2011. The link-offset-scale mechanism for improving the usability of touch screen displays on the web. In Proceedings of the 13th IFIP TC 13 international conference on Human-computer interaction - Volume Part III (INTERACT'11), Pedro Campos, Nuno Nunes, Nicholas Graham, Joaquim Jorge, and Philippe Palanque (Eds.), Vol. Part III. Springer-Verlag, Berlin, Heidelberg, 356-372.

[W3C 2013] World Wide Web Consortium (2013). WCAG 2.0.

http://www.w3.org/TR/WCAG20/

[W3C 1999]. World Wide Web Consortium (1999). HTML 4.01 Specification. http://www.w3.org/TR/html4/ 